\title{
Efficient Mapping of High Order Basis Sets for Unbounded Domains
}

\author{
Faisal Mumtaz ${ }^{1, *}$ and Fahhad H. Alharbi ${ }^{1,2}$ \\ ${ }^{1}$ College of Science and Engineering, Hamad Bin Khalifa University, Doha, Qatar. \\ 2 Qatar Environment \& Energy Research Institute (QEERI), Hamad Bin Khalifa \\ University, Doha, Qatar.
}

Received 16 March 2017; Accepted (in revised version) 31 July 2017

\begin{abstract}
Many physical problems involve unbounded domains where the physical quantities vanish at infinities. Numerically, this has been handled using different techniques such as domain truncation, approximations using infinitely extended and vanishing basis sets, and mapping bounded basis sets using some coordinate transformations. Each technique has its own advantages and disadvantages. Yet, approximating simultaneously and efficiently a wide range of decaying rates has persisted as major challenge. Also, coordinate transformation, if not carefully implemented, can result in non-orthogonal mapped basis sets. In this work, we revisited this issue with an emphasize on designing appropriate transformations using sine series as basis set. The transformations maintain both the orthogonality and the efficiency. Furthermore, using simple basis set (sine function) help avoid the expensive numerical integrations. In the calculations, four types of physically recurring decaying behaviors are considered, which are: non-oscillating and oscillating exponential decays, and non-oscillating and oscillating algebraic decays. The results and the analyses show that properly designed high-order mapped basis sets can be efficient tools to handle challenging physical problems on unbounded domains. Decay rate ranges as large of 6 orders of magnitudes can be approximated efficiently and concurrently.
\end{abstract}

AMS subject classifications: 65D99, 65M70

Key words: Mapped basis sets, unbounded domain, spectral method.

\section{Introduction}

As known, physical phenomena are modeled and represented mathematically by sets of differential equations with appropriate conditions (initial and/or boundary conditions).

*Corresponding author. Email addresses: faimumtaz@hbku.edu.qa (F. Mumtaz), falharbi@hbku.edu.qa (F. H. Alharbi) 
A small number of these differential equations can be solved analytically and hence numerical methods are routinely used to solve such problems. Therefore, numerous methods have been developed accordingly. They can be categorized into two general classes, namely, mesh-based and mesh-free methods [1-8]. In mesh-based methods, the computational window is spatially discretized (meshed) in a priori. The resulted predefined mesh is then utilized using low-order and localized basis sets to solve the considered differential equations in different ways [8]. The most commonly used mesh-based numerical methods are finite different methods (FDM) and finite element methods (FEM). Obviously, these methods scale up exponentially with the space dimension $(D)$; i.e. $\mathcal{O}\left(N^{D}\right)$. So, they become computationally very expensive for $D>2[5,9,10]$. However, for highly sparse matrices, better scaling can be achieved by further exploitation of the sparsity and by imposing locality to reduce the number of required operations. For example, in the sparse grid method, a computational cost of $\mathcal{O}\left(N(\log N)^{D-1}\right)$ can be achieved $[9,11,12]$. The second class is meshfree methods, where the unknown quantities are approximated by high order basis sets without the need for the mesh $[1,7]$. The most known techniques of this family are the various spectral and pseudospectral methods $[2,7,13]$ including Galerkin methods.

Recently, spectral methods (SM) have gained more attention and implementation primarily because of its high-order nature, which would result in high level of accuracy with less required computational resources (i.e. time and memory). This is because a considerable portion of the formulation is handled analytically $[6,7,14]$. In these methods, the real-space solution of a differential equation is presented in terms of a sum of certain basis or trial functions [15]. The basis functions are chosen to satisfy the constraints and needed conditions $[2,6,16-20]$. The methods then follow the general steps of weighted residual methods (WRM) where various weight (test) functions can be used. For more details about spectral methods and their applications, we refer the reader to more dedicated sources $[1,2,7,13,15,21]$.

The spectral methods have been implemented successfully for many challenging problems in bounded domains $[2,7,11,22]$. Basically, this is due to the finite computational domain and the abundance of convenient orthogonal basis sets for such bounded domains. More development is needed to match that for unbounded domains $[12,14,16,17,21]$. Actually, some numerical techniques have been used to apply SM for unbounded domains; but, with some persistent challenges. Among the commonly used techniques are the domain truncation, implementation of basis functions that are intrinsically unbounded, and coordinate transformation (mapping) $[12,14,16,17,21,23]$.

Domain truncation is one of the most commonly used techniques in FDM and FEM, where the infinite physical domain is represented by a finite computational window. Consequently, it will be inevitable to have a truncation error, which is reduced by increasing the size of the computation window. However, the larger computational window requires an accordingly larger number of mesh points. This can be mitigated by adopting nonuniform meshing and boundary layers. The second approach is to use functions like sinc and Hermite polynomials as basis sets. The computational domain 
of these functions extends to infinities and hence can be used for solving the physical problems involving unbounded domains. In the third approach, change of coordinates, the unbounded domain is converted into a bounded domain by using some appropriate transformation function. Conceptually, there are unlimited ways to map an unbounded domain to a bounded one. However, although, the concept of mapping is very straight forward, there are many aspects that should be considered to ensure the efficiency of a numerical method. First, orthogonality should be preserved in both physical and computational spaces $[24,25]$. Also, it is important to observe how the mapping grows near the edge of the mapped domain. Different families of mapping (logarithmic mapping, algebraic mapping, exponential mapping etc) exist depending upon the rate of growth of function near the edge of the domain [2]. Moreover and from a practical perspective, the mapping should not complicate significantly the differential equations and should allow analytical integration $[13,26]$. In addition to this, there are many physical problems that have a wide range of decay rates and multi-harmonics that the mapping should be able to account for concurrently. Sinc and Hermite polynomials fail for such problems $[6,14,16,19,26-29]$.

The concept of mapping in not new. It has been implemented successfully to convert unbounded domain to bounded one and vice versa [12,14, 16, 17, 21, 23, 30-32]. For example, Grosch and Orszag, in 1977, used mapping to transform semi-infinite domain $[0, \infty)$ to $(0,1)$ using exponential and algebraic mapping, and the resulting transformation functions are used to solve PDEs by approximating the solution using Chebyshev polynomials [30]. Boyd, in 1982, performed a comparison of domain truncation with algebraic and exponential mapping for the problems involving semi-infinite domain [31], and later, in 1987, implemented the mapping to solve ODEs using mapped Chebyshev polynomials [32]. In 2012, Shen proposed a generalized method to design transformation functions to transform $(-\infty, \infty)$ to $(-1,1)$ using algebraic mappings, and then used Chebyshev interpolation to solve the differential equations [12].

In this work, a general approach to design an efficient mapping based on Shen earlier work $[12,16]$ is implemented. The proposed approach can be implemented to physical problems that extend toward infinities where the involved physical quantities decay smoothly. The approach ensures orthogonality in both physical and computational spaces. Maintaining orthogonality simplifies the calculations significantly, and hence increasing the computational efficiency as shown later in the paper. More importantly, the approach approximates efficiently various type of decaying functions with a wide range of decay rates by appropriate choices of mapping and using simple functions like sine series as basis sets. Using simple basis sets helps in simplifying the implementation and in obtaining analytical integration used for solving ODEs and PDEs, and hence increasing computational efficiency.

The basis set used in this paper is the mapped sine series. The physical unbounded domain $(-\infty, \infty)$ is mapped to a computational bounded domain $(0,1)$. The mapping is tested on four different sets of decaying functions; namely, algebraic decay without and with oscillation, and exponential decay without and with oscillation, where a very 
wide range of decaying rates are used. It is shown that the appropriate transformations effectively approximate these types of functions. The range of decays that can be approximated efficiently is larger than 6 orders of magnitude. A comparison of the approximations and their efficiencies for the considered decaying functions is presented.

\section{Formulations}

\subsection{Coordinate transformation using mapped basis set}

In this section, the formulation used to develop efficient mappings from unbounded domain to a bounded one is presented. We focus on the one-dimensional (1D) case, which can be extended to any high-dimension problems by proper tensoral construction. Such mappings shall enrich the possibilities of efficient basis sets that can deal with the original unbounded domain. The transformation function is defined as:

$$
x=h(u), \quad u=h^{-1}(x)=g(x),
$$

where $x \in(-\infty, \infty)$ and $u \in(a, b)$ are the original physical space and the computational space variables, respectively. From basic calculus, the relation between the derivatives in both spaces is

$$
\frac{d}{d x}=\frac{1}{h^{\prime}(u)} \frac{d}{d u}
$$

where $h^{\prime}(u)=\frac{d x}{d u}$. There are an infinite possibilities for such transformation $[2,12,14,16]$. Let $\left\{P_{k}(u)\right\}$ be an orthogonal basis set with some weight function $w(u)$, then, the targeted form of the mapped basis functions is

$$
f_{k}(x)=P_{k}(g(x)) \mu(g(x)),
$$

where $\mu(u)$ is an auxiliary function. To satisfy the vanishing boundary conditions at $\infty$, either $P_{k}(g(x)), \mu(g(x))$ or both must vanish as $x \rightarrow \pm \infty$. In this work, we seek $\mu(g(x))$ that vanish at infinities. In order to maintain the orthogonality of $\left\{f_{k}(x)\right\}$, the following relationship must be satisfied:

$$
\int_{-\infty}^{\infty} f_{k}(x) f_{l}(x) d x=\delta_{k l}
$$

By applying the coordinate transformation $x \rightarrow u$, the orthogonality relation becomes

$$
\int_{a}^{b} P_{k}(u) P_{l}(u) \mu^{2}(u) \frac{d x}{d u} d u=\delta_{k l}
$$

where $\mu(u)$ is the mapped auxiliary function which vanishes at the boundaries of the domain $(a, b)$. Since $\left\{P_{k}(u)\right\}$ is an orthogonal basis set with some weight function $w(u)$, its orthogonality relationship in the computational space (i.e. $u \in(a, b))$ is

$$
\int_{a}^{b} P_{k}(u) P_{l}(u) w(u) d u=c_{k} \delta_{k l} .
$$


From Eq. (2.5) and Eq. (2.6), we see that the following relation must be satisfied to maintain the orthogonality in the physical space as well:

$$
\mu^{2}(u) \frac{d x}{d u}=\frac{w(u)}{c_{k}}
$$

In this paper, the sine series is used as the basis set in the computational space (i.e. $\left.P_{k}(u)=\sin (k \pi u)\right)$ and hence the associated weight function is $w(u)=1, c_{k}=1 / 2$, and $u \in(0,1)$. By using this and rearranging Eq. (2.7), we get

$$
\frac{d x}{d u}=\frac{w(u)}{\mu^{2}(u) c_{k}}=\frac{2}{\mu^{2}(u)}
$$

which illustrates that $\mu(u)$ is a key in designing transformations. It is actually the mean exploited in this paper. Beside efficient approximation of various forms of decaying functions with wide ranges of decaying rates, we seek transformations that can allow analytical integrations. As we use trigonometric basis set, it is reasonable to seek trigonometric $\mu(u)$ as well. Thus, the first considered family is

$$
\mu_{\mathbb{T}, r}(u)=\frac{\sin (\pi u)^{r / 2}}{\sqrt{L}}, \quad r \in \mathbb{N}_{1}
$$

Another transformation family, which is commonly used, is given by

$$
\mu_{\mathbb{A}, r}(u)=\frac{[u(1-u)]^{\frac{r}{4}}}{\sqrt{L}}, \quad r \in \mathbb{N}_{1} .
$$

It is used here for comparison purposes. In both cases, $L$ is used for scaling. As shown by Alharbi [6], the scaling is merely a unit of a scale normalization. It does not expand the range of decaying rates that are covered. The range will be only shifted. Thus, in this work, we use $L=1$.

Fig. 1 shows the behavior of both $\mu_{\mathbb{T}, r}$ and normalized $\mu_{\mathbb{A}, r}$ for various values of $r$. The behavior at the edges of the domain plays a critical role in the approximation efficiency which is depicted in the results. The boundaries 0 and 1 represent the behavior at $-\infty$ and $\infty$, respectively. As the value of $r$ increases, the decay behavior of $\mu_{r}$ becomes smoother allowing a wider range of decay rates to be approximated efficiently. Furthermore, for same values of $r, \mu_{\mathbb{T}, r}$ gives better smoothing when compared with $\mu_{\mathbb{A}, r}$, hence providing a better approximation efficiency over a wider range of decay behaviors. For the transformations associated with $\mu_{\mathbb{T}, r}$, we use $r=1: 6$ in this paper. Table 1 and Table 2 list the used transformations $x(u)$ in this paper. The square brackets are the notations to be used in the remaining of the paper to designate the different transformations. 


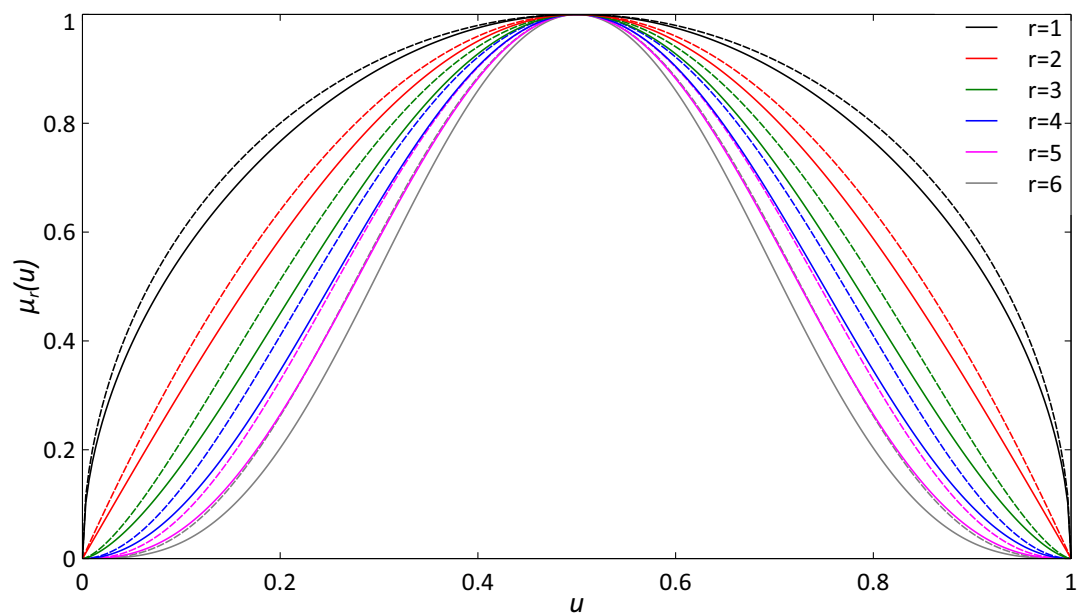

Figure 1: Behavior of both $\mu_{\mathbb{T}, r}$ (solid lines) and normalized $\mu_{\mathbb{A}, r}$ (dashed lines) for various values of $r$.

Table 1: The used trigonometric transformations $x(u)$ to map $x \in(-\infty, \infty)$ to $u \in(0,1)(L=1)$.

\begin{tabular}{||lll||}
\hline$r$ & Notation & $x(u)$ using $\mu_{\mathbb{T}, r}$ \\
\hline 1 & {$\left[T_{1}\right]$} & $\frac{2}{\pi} \log \left[\tan \left(\frac{\pi u}{2}\right)\right]$ \\
\hline 2 & {$\left[T_{2}\right]$} & $-\frac{2}{\pi} \cot (\pi u)$ \\
\hline 3 & {$\left[T_{3}\right]$} & $-\frac{1}{4 \pi} \csc ^{2}\left(\frac{\pi u}{2}\right)+\frac{1}{4 \pi} \sec ^{2}\left(\frac{\pi u}{2}\right)+\frac{1}{\pi} \log \left[\tan \left(\frac{\pi u}{2}\right)\right]$ \\
\hline 4 & {$\left[T_{4}\right]$} & $-\frac{2}{3 \pi} \cot (\pi u)\left[\csc ^{2}(\pi u)+2\right]$ \\
\hline 5 & {$\left[T_{5}\right]$} & $\frac{-1}{32 \pi}\left[\csc 4\left(\frac{\pi u}{2}\right)+6 \csc ^{2}\left(\frac{\pi u}{2}\right)-\sec ^{4}\left(\frac{\pi u}{2}\right)-6 \sec ^{2}\left(\frac{\pi u}{2}\right)-24 \log \left[\tan \left(\frac{\pi u}{2}\right)\right]\right]$ \\
\hline 6 & {$\left[T_{6}\right]$} & $-\frac{2}{15 \pi} \cot (\pi u)\left[3 \csc ^{4}(\pi u)+4 \csc ^{2}(\pi u)+8\right]$ \\
\hline
\end{tabular}

Table 2: The used algebraic transformations $x(u)$ to map $x \in(-\infty, \infty)$ to $u \in(0,1)(L=1)$.

\begin{tabular}{||lcl||}
\hline$r$ & Notation & $x(u)$ using $\mu_{\mathbb{A}, r}$ \\
\hline 1 & {$\left[A_{1}\right]$} & $-4 \tanh ^{-1}(1-2 u)$ \\
\hline 2 & {$\left[A_{2}\right]$} & $\frac{2}{(1-u)}-\frac{2}{u}-8 \tanh ^{-1}(1-2 u)$ \\
\hline
\end{tabular}




\subsection{Approximations of functions in unbounded domains}

The mapped basis set are used then to approximate the functions in unbounded domains where the functions vanish at infinities. So, the linear space of approximation $\Phi_{N}=\operatorname{span}\left\{f_{k}(x)\right\}$ is defined by

$$
\Phi_{N}=\left\{\phi: \phi(x)=\sum_{k=1}^{N} a_{k} f_{k}(x)=\sum_{k=1}^{N} a_{k} P_{k}(g(x)) \mu(g(x))\right\} .
$$

Given a function $\mathbb{S}(x)$, which is to be approximated where $x \in(-\infty, \infty)$. Then,

$$
\mathrm{S}(x) \approx \sum_{k=0}^{N} a_{k} P_{k}(g(x)) \mu(g(x)),
$$

where

$$
\begin{aligned}
a_{k} & =\int_{-\infty}^{\infty} \mathbf{S}(x) P_{k}(g(x)) \mu(g(x)) d x \\
& =\frac{1}{c_{k}} \int_{a}^{b} \frac{\mathbb{S}(x(u)) P_{k}(u) w(u)}{\mu(u)} d u .
\end{aligned}
$$

As we use sine series for the approximation in the computational domain, then $a_{k}$ is simply:

$$
a_{k}=\frac{1}{2} \int_{0}^{1} \frac{\sin (k \pi u) S(x(u))}{\mu(u)} d u
$$

\subsection{Implementation to solve differential equations in unbounded domains}

In this subsection, the proposed approximation is implemented to solve differential equations, where the solutions of these differential equations extend toward infinities and die smoothly. Since the main scope of this paper is using the mapped basis sets to approximate functions in unbounded domain, for differential equations, only simple 1D differential equations problems are presented for illustrations. The considered problems take generally the following form:

$$
\hat{\mathcal{L}} y(x)=\mathcal{F}(x),
$$

where $\hat{\mathcal{L}}$ is a differential operator, operating on $y(x)$, and $\mathcal{F}(x)$ is the force function. We assume that the solution can be expanded using the mapped basis set; so,

$$
\tilde{y}(x)=\sum_{l=1}^{N} a_{l} f_{l}(x),
$$

where $f_{l}(x)$ is given by Eq. (2.3). By inserting Eq. (2.16) in Eq. (2.15) and by calculating the inner products $\left(f_{k}(x) \mid \sum_{l=1}^{N} a_{l} \hat{\mathcal{L}} f_{l}(x)\right)$ and $\left(f_{k}(x) \mid \mathcal{F}(x)\right)$, we get a set of linear equations which can be written in matrix form as:

$$
\mathrm{La}=\boldsymbol{\Gamma},
$$


where $\mathbf{a}$ is the vector of unknown coefficients, $\mathbf{L}$ is the resulting matrix from the inner product with the left-hand side, and $\Gamma$ is the resulting vector from the inner product with the right-hand side. Once $\mathbf{L}$ and $\Gamma$ are calculated, the unknown coefficients are be then calculated directly and the final solution can be computed using Eq. (2.16).

\section{Results and discussions}

This section presents the approximation accuracy of four types of decaying functions, $\mathbb{S}(x)$, with a wide range of decay rates. The considered functions are given in Table 3 . The parameter $\alpha$ in the decaying functions represents the decay rate, whereas $k$ incorporates for the oscillation. As we consider unbounded domains, $\mathbb{S}(x)$ extends towards infinities (i.e. $x \in(-\infty, \infty))$. The eight transformations shown in Table 1 and Table 2 are used with the given basis set. The transformation functions used are given in Table 1 and Table 2 where as the basis functions are according to Eq. (2.11) where $P_{n}(u) \equiv \sin (n \pi u)$. MATLAB 2016b is used along with ADVANPIX multi-precision toolbox to evaluate the proposed numerical method. ADVANPIX allows working beyond the double precision allowed in basic MATLAB. The results and analyses are based on the resulted errors, which are quantified by their supremum norms $L_{\infty}$. This is because the aim is to improve the approximation all over the considered domain and hence we need to minimize the peak error.

Table 3: The considered decaying functions.

\begin{tabular}{||ll||}
\hline Exponential decay without oscillation & $\mathrm{S}(x)=e^{-\alpha x^{2}}$ \\
Exponential decay with oscillation at infinity & $\mathrm{S}(x)=\cos (k x) e^{-\alpha x^{2}}$ \\
Algebraic decay without oscillation & $\mathrm{S}(x)=\left(1+x^{2}\right)^{-\alpha}$ \\
Algebraic decay with oscillation at infinity & $\mathrm{S}(x)=\frac{\cos (k x)}{\left(1+x^{2}\right)^{\alpha}}$ \\
\hline
\end{tabular}

In the first analysis, the approximation accuracy is presented for several cases. These cases are classified depending on the number of basis used $(N=100$ or $N=200)$ and the type of decaying function (exponential or algebraic). Case I and Case II cover the approximation accuracy results for the exponentially decaying functions for $N=100$ and $N=200$, respectively. Case III and Case IV cover the approximation accuracy results for the algebraically decaying functions for $N=100$ and $N=200$, respectively. Results for the Case I are shown in Fig. 2. The graph shows the approximation errors of exponentially decaying functions vs. the decay rate $(\alpha)$, which is varied between $10^{-4}$ and $10^{5}$. In this figure and the next three ones, subfigure (a) shows the plot of decay without oscillation, whereas subfigures (b), (c) and (d) show the plots for decay with oscillation when $k$ is set to 1, 2 and 5, respectively. Results for the Case II are depicted in Fig. 3. In this case, the 


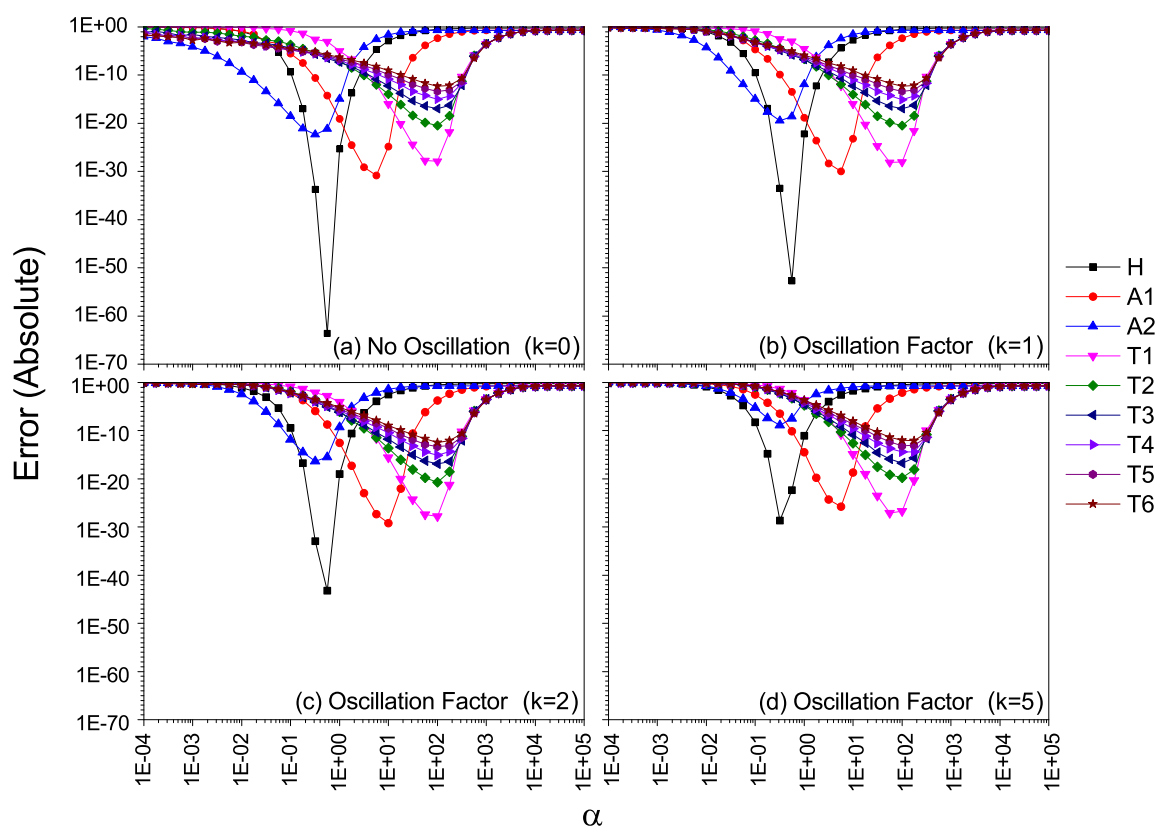

Figure 2: Case I: Exponential decay with and without oscillation: error vs. $\alpha$ for $N=100$.

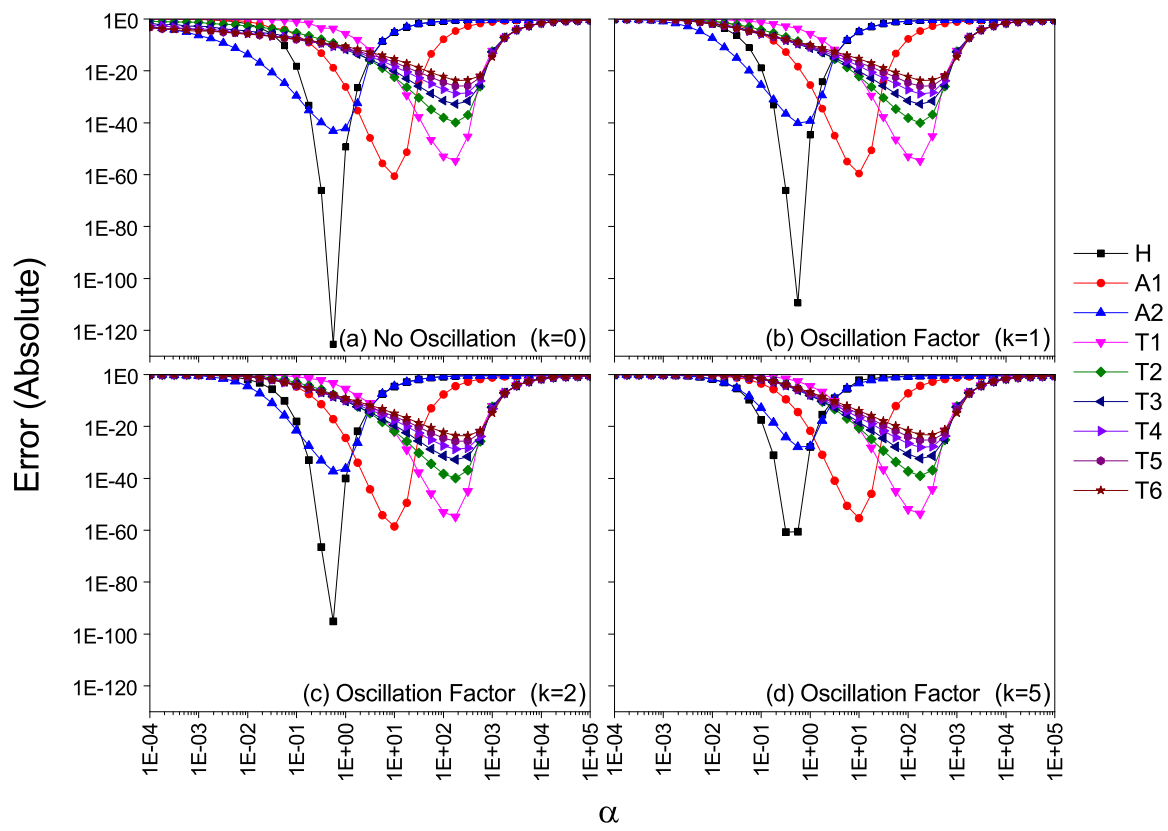

Figure 3: Case II: Exponential decay with and without oscillation: error vs. $\alpha$ for $N=200$. 
number of used basis functions is increased to 200. In both cases (Case I and Case II), the new mapped basis set is compared to the Hermite approximation.

As can be seen from Fig. 2 and Fig. 3, i.e. Case I and Case II, respectively, the Hermite approximation is only efficient for a very small range of decays around $\alpha=0.5$. On the contrary, other transformations give wider ranges of $\alpha$ that can be approximated efficiently. This is quantified and discussed shortly. Also, it can be seen that the optimum range of each transformation is differently centered. However, this can be shifted using a scaling. For algebraic transformation, the centers varied significantly where $r$ is changed from 1 to 2, while those resulted from trigonometric transformations are almost the same as $r$ increased from 1 to 6 . However, the error increases with $r$. Yet, the approximations are still efficient where the errors are less than $10^{-10}$.

The next two cases (Case III and Case IV) are for algebraically decaying functions where the approximation accuracy is also tested. In both cases $\alpha$ is varied between $10^{-2}$ and $10^{5}$. As mentioned above, Case III covers the results for algebraically decaying functions where the number of basis used are 100. Plots from the results of Case III are shown in Fig. 4 which shows the approximation errors of algebraically decaying functions vs. the decay rate $(\alpha)$. Results for Case IV are depicted in Fig. 5. Here, the number of used basis functions is increased to 200. In both cases (Case III and Case IV), the algebraic transformations and Hermite approximation result in very poor approximations. On the contrast, the trigonometric transformations provide very accurate approximations and for very wide range of $\alpha$ where the errors are less than $10^{-10}$.

The second analysis is for the implementation of the proposed basis sets to solve differential equations. As aforementioned, the focus of this paper is approximation of functions in unbounded domain. Hence, we have considered here simple $1^{\text {st }}$ and $2^{\text {nd }}$ order ODEs in 1D. By setting the differential operator $\hat{\mathcal{L}}=\frac{d y}{d x}$, and the force function $\mathcal{F}(x)=-2 \alpha x e^{-\alpha x^{2}}$, the differential equation becomes

$$
\frac{d y}{d x}=-2 \alpha x e^{-\alpha x^{2}}
$$

and its solution is

$$
y(x)=e^{-\alpha x^{2}} .
$$

Any transformation function can be used to solve Eq. (3.1). Here, we consider trigonometric transformation $\left[T_{1}\right]$. In this case, the inner product $\left(f_{k}(x) \mid \sum_{l=1}^{N} \frac{d f_{l}(x)}{d x}\right)$ can be found analytically (an advantage of using trigonometric transformation and bases functions) and the resulted matrix is $\mathbf{L}=\left[L_{k l}\right]$ where

$$
L_{k l}=\frac{\pi}{8}\left[(1-2 l) \delta_{k, l-1}+(1+2 l) \delta_{k, l+1}\right],
$$

where $\delta_{i j}$ is Kronecker delta function while $\boldsymbol{\Gamma}=\left[\Gamma_{k}\right]$ can be calculated numerically where

$$
\Gamma_{k}=\int_{0}^{1} 2 \mathcal{F}(h(u)) \frac{\sin (k \pi u)}{\sin (\pi u)^{1 / 2}} d u .
$$




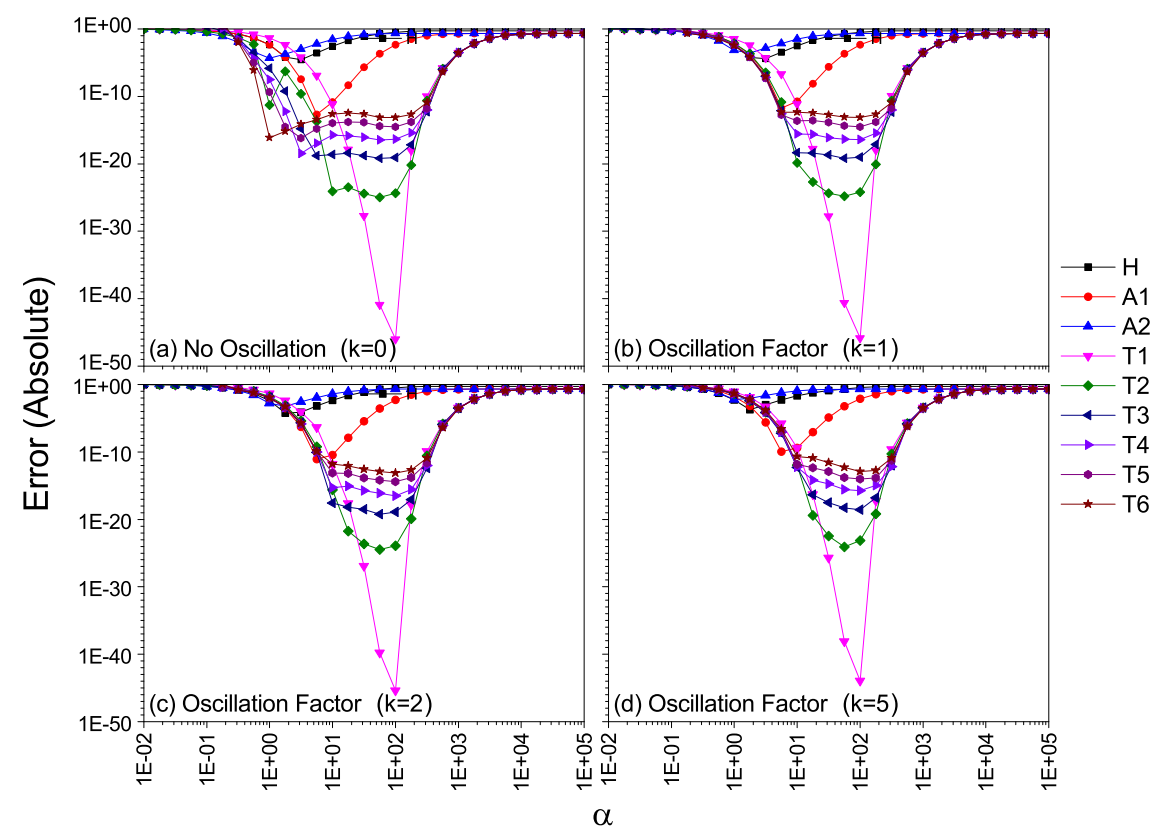

Figure 4: Case III: Algebraic decay with and without oscillation: error vs. $\alpha$ for $N=100$.

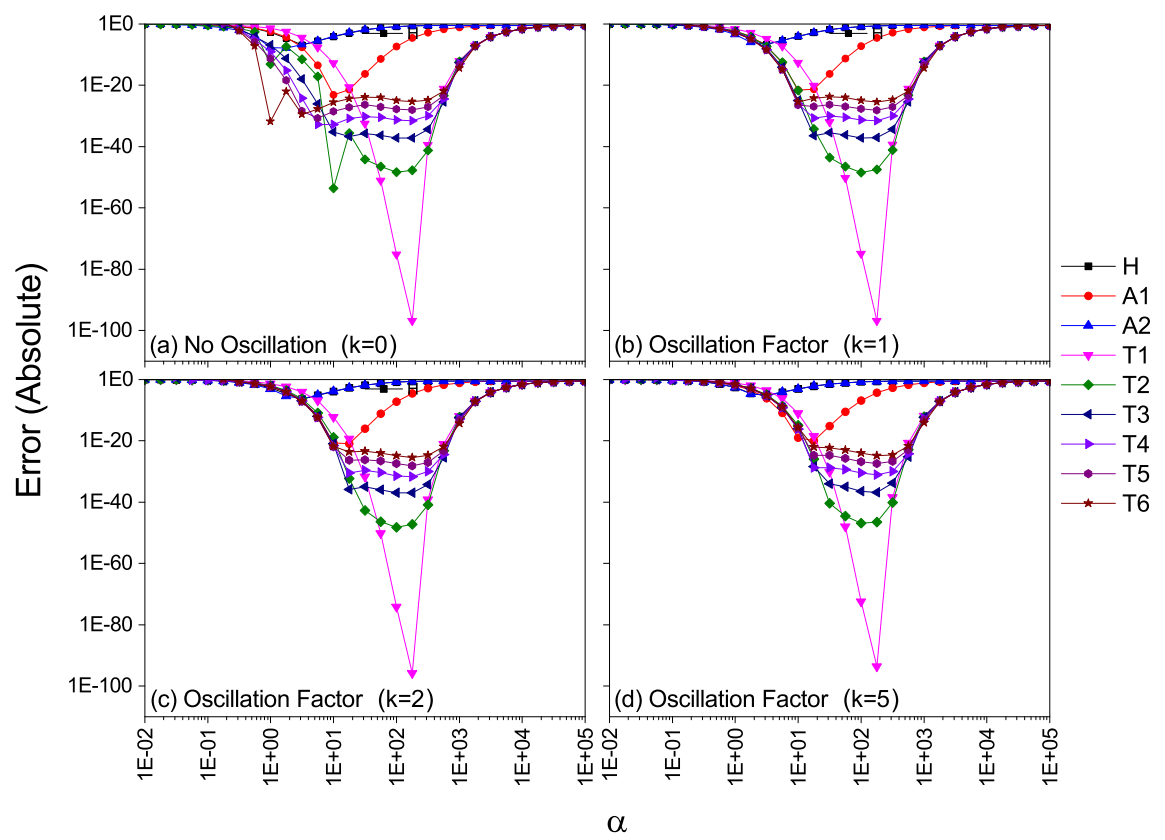

Figure 5: Case IV: Algebraic decay with and without oscillation: error vs. $\alpha$ for $N=200$. 
(a)

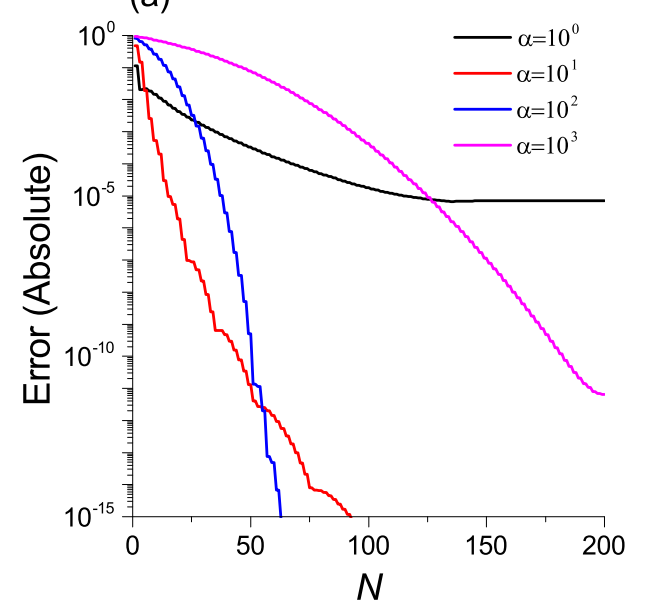

(b)

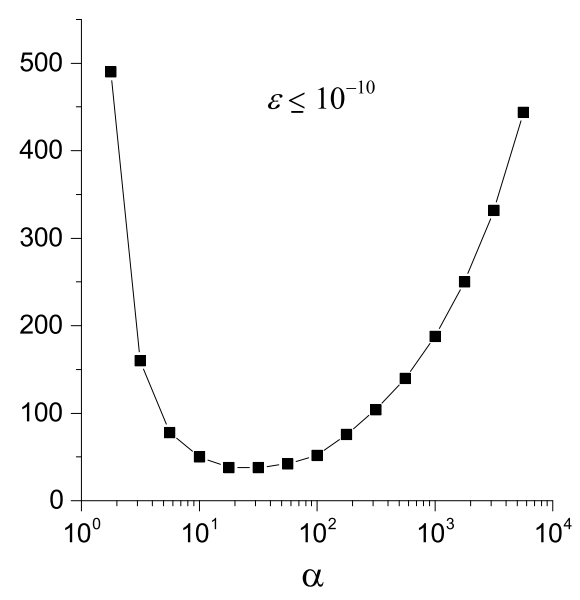

Figure 6: The error of the numerical solutions of ODE (Eq. (3.1)) considering various decay rates $(\alpha)$. (a) The errors of the numerical solutions vs. $N$ for various values of $\alpha$. (b) $N$ needed to achieve $\varepsilon \leq 10^{-10}$ for various values of $\alpha$.

Fig. 6 shows the errors of the numerical solutions for various values of $\alpha$. Fig. 6(a) shows how the numerical error decreases by increasing $N$, and how the decay rate $(\alpha)$ affects the error. As can be seen from the results, for very low decay rates and very high decay rates, a higher number of basis functions are needed to achieve a better accuracy. Whereas, for the range $(2<\alpha<1000)$, a good accuracy can be obtained with relatively less number of basis function. The rate of decay clearly affects the number of basis functions needed to achieve a particular accuracy. This is depicted in Fig. 6(b), which shows the number of basis functions required to find the solution within the accuracy of $10^{-10}$ for a particular $\alpha$.

The same approach can be extended to higher order differential equations. For example, consider the following $2^{\text {nd }}$ order ODE:

$$
\frac{d^{2} y}{d x^{2}}=2 \alpha e^{-\alpha x^{2}}\left(2 \alpha x^{2}-1\right)
$$

where the exact solution of Eq. (3.5) is given by Eq. (3.2). The approximate solution is expanded as in Eq. (2.16). In this case, the operator matrix $\mathbf{L}$ for the second derivative is simply the square of the operator matrix for the first derivative (Eq. (3.3)) due to the orthonormality of the used bases sets. The results of $2^{\text {nd }}$ order ODE are shown in Fig. 7, where the Fig. 7(a) shows the graph of numerical error vs. $N$ for various value of $\alpha$. Again, for very low or high values of $\alpha$, higher number of basis functions are needed of achieve a particular accuracy. This can be seen clearly in Fig. 7(b), which shows the number of basis functions required to find the solution within the accuracy of $10^{-10}$ for a particular $\alpha$. It is clear that for $2<\alpha<1000$, an accuracy of $10^{-10}$ can be achieved using relatively less number of basis functions. 
(a)

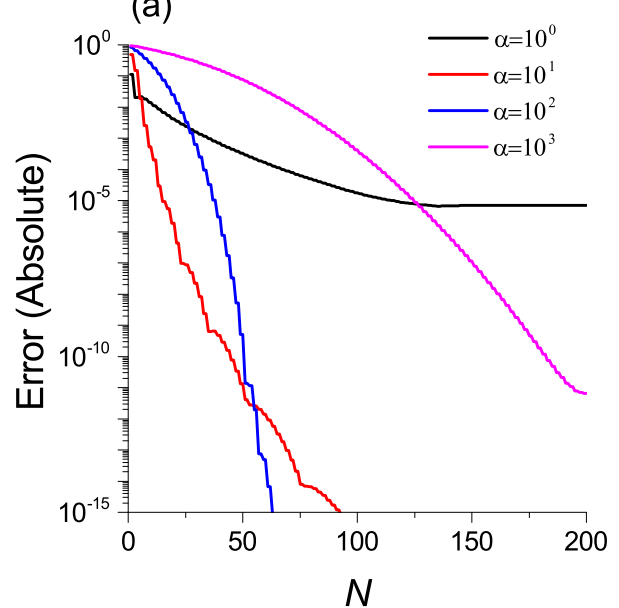

(b)

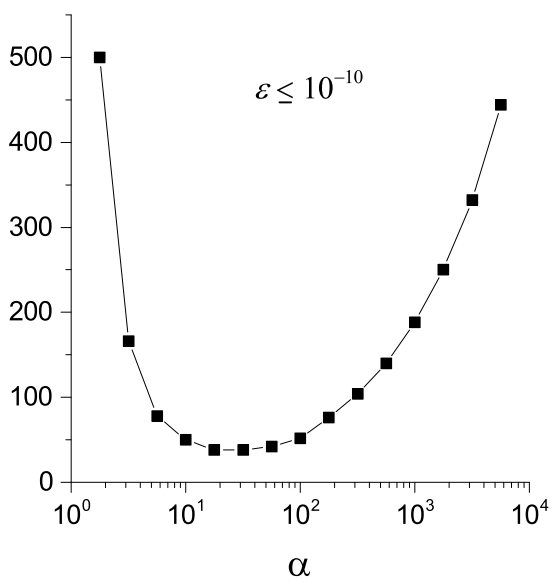

Figure 7: The error of the numerical solutions of ODE (Eq. (3.5)) considering various decay rates $(\alpha)$. (a) The errors of the numerical solutions vs. $N$ for various values of $\alpha$. (b) $N$ needed to achieve $\varepsilon \leq 10^{-10}$ for various values of $\alpha$.

The last analysis is to quantify the range of $\alpha$ that can be approximated efficiently, for a fixed number of basis functions, we check the values of $\alpha$ that can be approximated with a target accuracy. The used target accuracy is $10^{-10}$ for all the cases. This is depicted in Fig. 8 and Fig. 9, where a comparison is provided for the order of magnitude (OM) for different transformations. The $\mathrm{OM}$ is a measure of how much wider ranges of $\alpha$ can be approximated accurately and efficiently, and this is the measure that is used in this analysis. The OM is computed for $N=(100,200,300$ and 500). An OM of greater than 6 can be achieved using $N=500$ in the case of exponential decay without oscillation (Fig. 8(a)) The approximation accuracy decreases with the increase in the oscillations. Still, a very good $\mathrm{OM}$ can be achieved using trigonometric transformations. Even with $k=5$, an $\mathrm{OM}$ of greater than 4 can be achieved in the case of exponentially decaying functions. In the case of algebraically decaying functions, the algebraic transformations and the Hermite approximation fail to achieve the target accuracy $\left(10^{-10}\right)$ in several cases as shown in Fig. 9. The approximation efficiency of Hermite and algebraic transformations become worse when the oscillation factor is increased to 5 . However, an OM of greater than 3 can still be achieved with the trigonometric transformations (Fig. 9(d)). From the results, it can be seen that all the transformation functions outmatch Hermite approximation in all the cases. Furthermore, the rate at which is the OM increases with the number of basis $(N)$ used is also higher when compared with Hermite.

The comparisons show that the proposed mappings are efficient, accurate and robust. Also, the results show that the proposed method dominates Hermite approximation in all the cases, and gives a very wide range of decays can be approximated accurately and efficiently. 


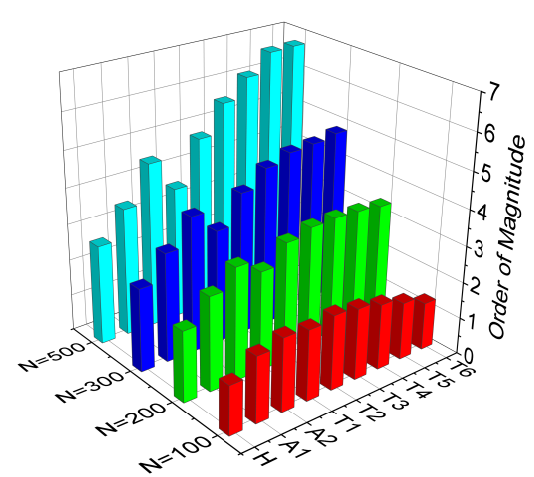

(a) No oscillation $(\mathrm{k}=0)$.

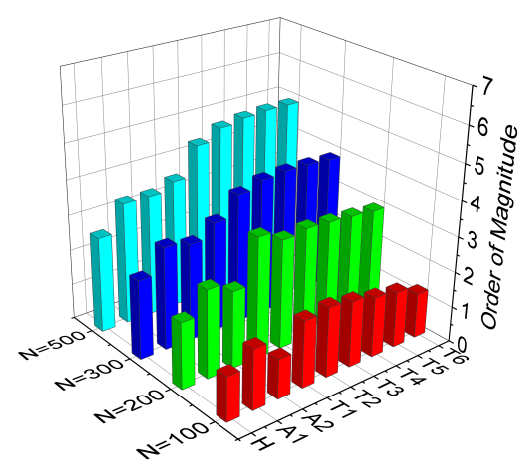

(c) Oscillation factor $(\mathrm{k}=2)$.

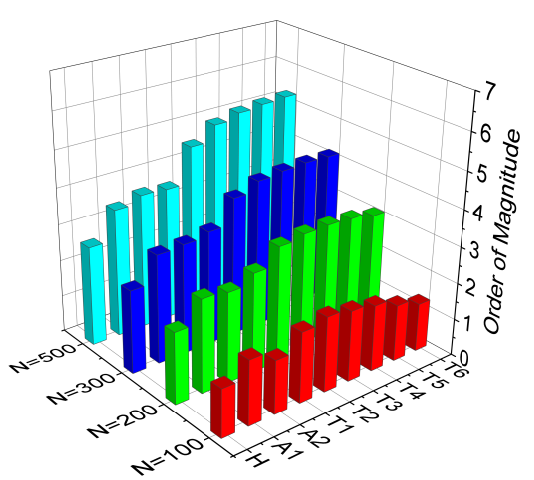

(b) Oscillation factor $(\mathrm{k}=1)$.

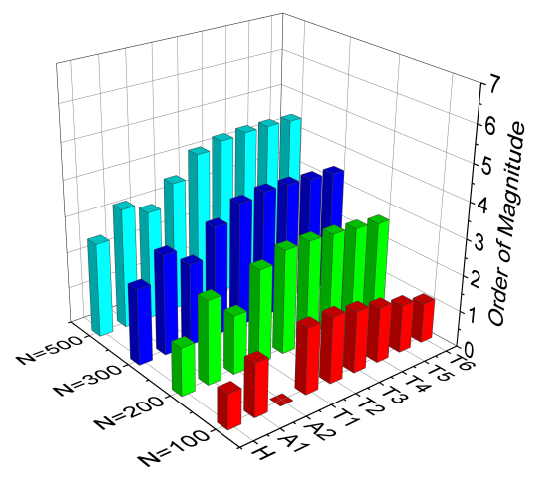

(d) Oscillation factor $(\mathrm{k}=5)$.

Figure 8: Order of magnitude for exponentially decaying functions.

\section{Conclusions}

In this paper, an efficient mapping is presented to solve the physical problems involving unbounded domains with vanishing boundary conditions. The mapping transforms the unbounded physical domain $(-\infty, \infty)$ to bounded computational domain $(0,1)$. Mapped sine series using general coordinate transformation for unbounded domains is used as the mapped basis set. The proposed scheme is implemented to approximate four sets of decaying functions with a wide range of decay rates. The decay functions extend to infinities where they die smoothly. Approximation accuracy of the proposed method is compared with Hermite approximation which is a standard choice for the problems extending to infinities. A very good accuracy can be achieved using a small number of basis functions for various decay rates. In addition to this, the order of magnitude is also used as a measure to assess the wide range of decay rate that can be approximated efficiently. Furthermore, the proposed approximation scheme is also implemented to solve differential equations, where the solutions of such differential equations decay smoothly towards infinities. The proposed approximation is implemented to solve $1^{\text {st }}$ and $2^{\text {nd }}$ order ODEs 


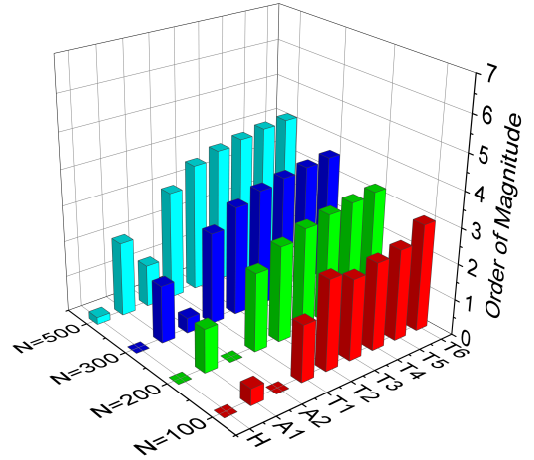

(a) No oscillation $(\mathrm{k}=0)$.

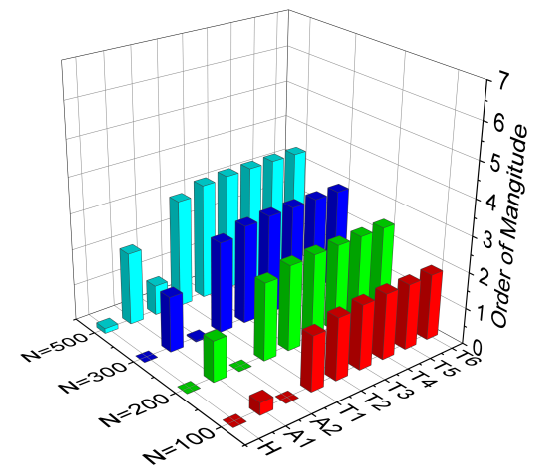

(c) Oscillation factor $(\mathrm{k}=2)$.

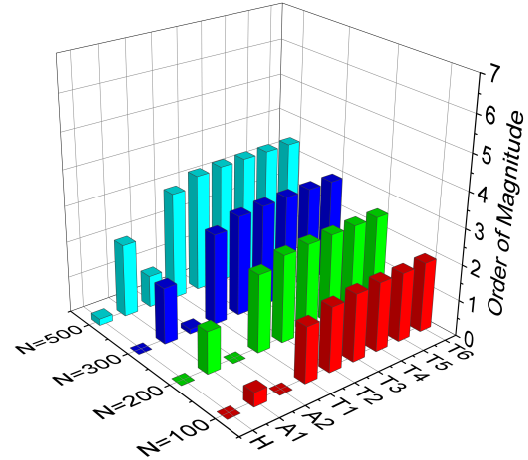

(b) Oscillation factor $(\mathrm{k}=1)$.

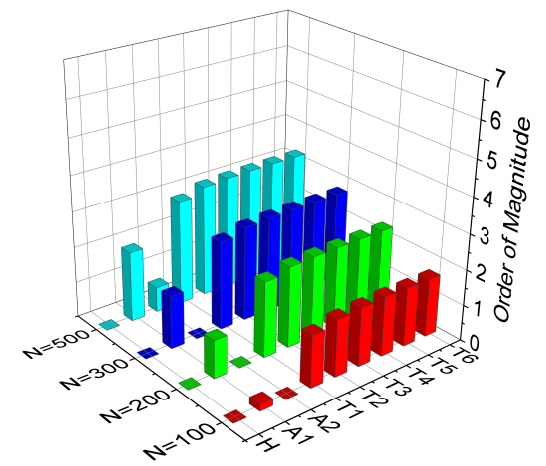

(d) Oscillation factor $(\mathrm{k}=5)$.

Figure 9: Order of magnitude for algebraically decaying functions.

in $1 \mathrm{D}$, and the approximate solution is compared with the exact solution. It has been shown that an accuracy of $10^{-10}$ can be achieved with a few number of basis functions for a wide range to decay rates. The results also show that the proposed method is efficient, accurate and outperforms Hermite approximation in all the cases, and it can be a useful tool to solve the physical problems involving unbounded domains.

\section{Acknowledgments}

The author would like to thank Qatar National Research Fund as the work is partially supported by NPRP 7-317-1-055.

\section{References}

[1] Jie Shen and Tao Tang. Spectral and high-order methods with applications. Mathematics monograph series. Science Press, 2006. 
[2] John P. Boyd. Chebyshev and Fourier Spectral Methods: Second Revised Edition. Dover Books on Mathematics. Courier Corporation, 2001.

[3] Xiong Zhang, Kang Zhu Song, Ming Wan Lu, and X Liu. Meshless methods based on collocation with radial basis functions. Computational Mechanics, 26(4):333-343, 2000.

[4] I Boztosun and A Charafi. An analysis of the linear advection-diffusion equation using mesh-free and mesh-dependent methods. Engineering Analysis with Boundary Elements, 26(10):889-895, 2002.

[5] Edward J Kansa, Ralph C Aldredge, and Leevan Ling. Numerical simulation of twodimensional combustion using mesh-free methods. Engineering Analysis with Boundary Elements, 33(7):940-950, 2009.

[6] Fahhad Alharbi. Comparative analysis of spectral methods in half-bounded domains; implementation of predefined exponential and laguerre basis sets to study planar dielectric and plasmonic waveguides. Optical and Quantum Electronics, 41(10):751-760, 2009.

[7] Philippe Grandclément and Jérôme Novak. Spectral methods for numerical relativity. Living Reviews in Relativity, 12(1):103 pages, 2009.

[8] Thomas L. Beck. Real-space mesh techniques in density-functional theory. Reviews of Modern Physics, 72:1041-1080, Oct 2000.

[9] Christoph Pflaum and Rainer Hartmann. A sparse grid discretization of the helmholtz equation with variable coefficients in high dimensions. SIAM Journal on Numerical Analysis, 54(4):2707-2727, 2016.

[10] Gary L Miller, Shang-Hua Teng, William Thurston, and Stephen A Vavasis. Geometric separators for finite-element meshes. SIAM Journal on Scientific Computing, 19(2):364-386, 1998.

[11] Jie Shen and Haijun Yu. Efficient spectral sparse grid methods and applications to highdimensional elliptic problems. SIAM Journal on Scientific Computing, 32(6):3228-3250, 2010.

[12] Jie Shen and Haijun Yu. Efficient spectral sparse grid methods and applications to highdimensional elliptic equations ii. unbounded domains. SIAM Journal on Scientific Computing, 34(2):A1141-A1164, 2012.

[13] C. Canuto, M.Y. Hussaini, A. Quarteroni, and T.A. Zang. Spectral methods: Fundamentals in single domains. Scientific Computation. Springer Berlin Heidelberg, 2007.

[14] Raka Jovanovic, Sabre Kais, and Fahhad H Alharbi. Efficient method for localized functions using domain transformation and fourier sine series. Molecular Physics, 112(5-6):762-769, 2014.

[15] Philippe Grandclement. Introduction to spectral methods. EAS Publications Series, 21:153180, 2006.

[16] Jie Shen and Li lian Wang. Some recent advances on spectral methods for unbounded domains. Communications in Computational Physics, 5(2):195-241, 2009.

[17] Ayşe Betül Koç and Aydın Kurnaz. A new kind of double chebyshev polynomial approximation on unbounded domains. Boundary Value Problems, 2013(1):10, 2013.

[18] Akil C Narayan and Jan S Hesthaven. A generalization of the wiener rational basis functions on infinite intervals, part iinumerical investigation. Journal of Computational and Applied Mathematics, 237(1):18-34, 2013.

[19] Fahhad H. Alharbi and Sabre Kais. Quantum criticality analysis by finite-size scaling and exponential basis sets. Physical Review E - Statistical, Nonlinear, and Soft Matter Physics, 87(4), 42013.

[20] Raka Jovanovic, Sabre Kais, and Fahhad H. Alharbi. Spectral method for solving the nonlinear thomas-fermi equation based on exponential functions. Journal of Applied Mathematics, 2014:168568, 2014. 
[21] BenYu Guo. Some progress in spectral methods. Science China Mathematics, 56(12):2411-2438, 2013.

[22] Bogdan Mihaila and Ioana Mihaila. Numerical approximations using chebyshev polynomial expansions: El-gendi's method revisited. Journal of Physics A: Mathematical and General, 35(3):731, 2002.

[23] Chao Zhang and Ben-yu Guo. Domain decomposition spectral method for mixed inhomogeneous boundary value problems of high order differential equations on unbounded domains. Journal of Scientific Computing, 53(2):451-480, 2012.

[24] Ben-yu Guo, Jie Shen, and Zhong-qing Wang. Chebyshev rational spectral and pseudospectral methods on a semi-infinite interval. International Journal for Numerical Methods in Engineering, 53(1):65-84, 2002.

[25] Zhong-Qing Wang and Ben-Yu Guo. A rational approximation and its applications to nonlinear partial differential equations on the whole line. Journal of Mathematical Analysis and Applications, 274(1):374-403, 2002.

[26] Fahhad Alharbi. Predefined exponential basis set for half-bounded multi domain spectral method. Applied Mathematics, 1:146-152, 2010.

[27] Fahhad Alharbi. Full-vectorial meshfree spectral method for optical-waveguide analysis. IEEE Photonics Journal, 5(1):6600315, 2013.

[28] Fahhad Alharbi and J Campbell Scott. Multi-domain spectral method for modal analysis of optical waveguide. Optical and quantum electronics, 41(8):583-597, 2009.

[29] Andreas Nold, Benjamin D. Goddard, Peter Yatsyshin, Nikos Savva, and Serafim Kalliadasis. Pseudospectral methods for density functional theory in bounded and unbounded domains. Journal of Computational Physics, 334:639-664, 2017.

[30] Chester E Grosch and Steven A Orszag. Numerical solution of problems in unbounded regions: coordinate transforms. Journal of Computational Physics, 25(3):273-295, 1977.

[31] John P Boyd. The optimization of convergence for chebyshev polynomial methods in an unbounded domain. Journal of computational physics, 45(1):43-79, 1982.

[32] John P Boyd. Spectral methods using rational basis functions on an infinite interval. Journal of Computational Physics, 69(1):112-142, 1987. 